\title{
Quality of Delivery Service at Public Health Facilities in Arba Minch District, Gamo Gofa Zone, Southern Ethiopia
}

\author{
Zeritu Dewana ${ }^{1,}$, Abebe Gebremariam², Misra Abdulahi ${ }^{2}$, Teshale Fikadu ${ }^{3}$, Wolde Facha ${ }^{3}$ \\ ${ }^{1}$ Department of Midwifery, Arba Minch Health Science College, Arba Minch, Ethiopia \\ ${ }^{2}$ Department of Population and Family Health, College of Public Health and Medical Sciences, Jimma University, Jimma, Ethiopia \\ ${ }^{3}$ School of Public Health, College of Health Sciences and Medicine, Wolaita Sodo University, Wolaita Sodo, Ethiopia
}

\section{Email address:}

dewanazeritu@gmail.com (Z. Dewana), abebe_gebremariam@yahoo.com (A. Gebremariam), misra_ab@yahoo.com (M. Abdulahi), fikaduteshale1@gmail.com (T. Fikadu),woldiefacha@gmail.com (W. Facha)

${ }^{*}$ Corresponding author

\section{To cite this article:}

Zeritu Dewana, Abebe Gebremariam, Misra Abdulahi, Teshale Fikadu, Wolde Facha. Quality of Delivery Service at Public Health Facilities in Arba Minch District, Gamo Gofa Zone, Southern Ethiopia. Journal of Gynecology and Obstetrics. Vol. 5, No. 2, 2017, pp. 31-36. doi: $10.11648 /$ j.jgo.20170502.12

Received: February 10, 2017; Accepted: March 7, 2017; Published: March 31, 2017

\begin{abstract}
Quality of delivery care service is an important aspect of maternal healthcare which enhance delivery services utilization by mothers. Assessing quality of delivery services is a global priority particularly in developing countries; however reports on this aspect are scarce, which calls for further study. Therefore, the objective of this study was to assess quality of delivery care at public health facilities in Arba Minch district, Gamo Gofa zone, Southern Ethiopia. A cross sectional facility based study was conducted among women who gave birth at public health facilities in this area. A total of nine health facilities, 27 key informants and 256 women were included in the study. Data were analyzed using SPSS 20.0 statistical software. The overall quality of delivery care was $54.06 \%$ and mother's satisfied on delivery care was $90.2 \%$. Shortage of some medical equipment, drugs and supplies, trained man power, few proper partograph records, good client provider interaction, good client satisfaction and low infection prevention practice was observed in our study. As a conclusion poor quality delivery service was observed at public health facilities. Thus, efforts should be made by government for improving facilities capacity and performance of care providers in order to improve the quality of delivery care.
\end{abstract}

Keywords: Delivery, Quality Care, Satisfaction, Delivery Service, Southern Ethiopia

\section{Introduction}

Donabedian defined quality care as "A care which is expected to maximize an inclusive measure of client welfare, after one has taken account of the balance of expected gains and losses that attend the process of care" with the following three distinct factors: the structure, the process, and the outcomes [1-3]. Structure is organizational factors that define the health system under which care is provided. It includes physical resources (infrastructure, equipment, drugs and supplies) and human resources (adequate, appropriately trained, motivated and supervised care providers). Process is the interactions between users and health care providers; it can be thought of as the actual delivery and receipt of care and outcome is the consequences of care [4]. Delivery of quality health services is central intervention to improve the health status of mothers and satisfy clients as a primary goal. It is provision of health services as per the standard at all levels of health facilities [5]. Globally an estimated 287,000 maternal deaths occur annually, 99\% occurred in developing countries, by concentrating in Sub-Saharan Africa (56\% of the total deaths globally) [6-8]. Ethiopia is one of the countries that have the highest maternal mortality rates in the world in which about seven women die during pregnancy, childbirth, or within two months of birth [9]. To reduce maternal deaths dramatically, all women need access to high quality delivery care with at least three key elements: skilled care at birth, emergency obstetric care in case of complications and a functioning referral system [6, 9]. Recent evaluation showed that progress in reduction of 
maternal mortality has been slow in Sub-Saharan Africa because of low quality of care, inadequate human resources, insufficient access to essential health technologies like essential equipments, drugs, supplies and financial barriers to care for the provision of safe delivery care [6, 10-12].

The country assessment also revealed that lack of infrastructure, shortage of human resource, lack of equipment, low partograph use and poor service quality during labor and delivery were observed at all levels of hospitals [13-15]. To address the issues, at national level, the government of Ethiopia has been implementing the health sector development program by strengthening Health Extension Program; training and deployment of skilled health professionals; accelerated construction and expansion of new health centers and hospitals; renovation and maintenance of existing hospitals and health centers; expansion of emergency obstetric care (EmOC), implementing quality management such as nursing standard, infection prevention and medical record standard; and establishing a well functional referral system [16-19]. Despite of these efforts still maternal morbidity and mortality was high and coverage of skilled birth attendant during delivery care was low. Thus, the purpose of this study was to assess quality of delivery care service at public health facilities in Arba Minch town and the surrounding district for the development and improvement of intervention strategies to reduce maternal morbidity and mortality.

\section{Methods and Materials}

\subsection{Study Design and Setting}

Facility-based cross sectional study was conducted in April, 2014 in Arba Minch town and the surrounding districts of Gamo Gofa zone, Southern Ethiopia. It is located $505 \mathrm{Km}$ south of Addis Ababa (the capital city of Ethiopia). The district had a total population of 300,057 for the year 2013/14 which was projected from 2007 Ethiopia Central Statistical Agency. The area has one hospital and eight health centers providing an estimated 3,876 delivery services annually.

\subsection{Sample Size and Sampling Procedure}

Both qualitative and quantitative methods of data collections were used. Sample size for quantitative data was calculated using Epi info version 7 by assuming the proportion properly recorded partograph according to standard as 20.7\% [21], 95\% confidence interval, $5 \%$ degree of precision, $10 \%$ possible non-response rate and with estimated annual delivery of 3876 . The final sample size of 261 was obtained and then allocated proportionally to all public health facilities based on annual average number of women who gave birth in each health facility. For qualitative study twenty seven in-depth interviews were conducted with purposively selected key informants (three from each health facility). They were head of health facilities, delivery ward team leaders and drug store keepers.

\subsection{Data Collection and Management}

Data were collected using structured questionnaire (which was initially prepared in English and translated into local language, then retranslated to check consistency) via face to face interview at exit. The instrument was pre-tested in 5\% of sample size in adjacent district public health facility. Nine data collectors who have diploma in midwifery after two days training were involved. Data were checked for completeness, then edited, coded and entered into Epi data version 3.1 and exported to SPSS 20.0 statistical software for analysis. Client satisfaction were assessed by 15 questions on a five point likert scale with score values ranging from 1 (very dissatisfied) to 5 (very satisfied).

\subsection{Data Analysis}

Factor analysis was employed for likert scale questions to facilitate treatment of variable as continuous for further analysis. Variables were reduced to one component to measure client satisfaction towards delivery care service with Cronbach's Alpha (reliability coefficient) value of greater than 0.7. For the overall satisfaction level, those who were satisfied in greater or equal to factor mean score of the items were categorized under "satisfied" and those who were satisfied in less than factor mean score of the items were categorized as "un satisfied". Descriptive statistics was computed for the study variables and data were presented using tables and figures. Variables with p-value less than 0.25 in bivariate analysis were included in a backward stepwise logistic regression procedure. Odds ratios with 95\% confidence intervals were calculated to determine the association between client satisfaction and independent variables. Model fitness was assessed using Hosmer and Lemeshow test $(\mathrm{p}=0.77)$. The resulting quality indices, which were weighted averages of indicators, expected to fall within 0 to 100 percent. The final summary scores developed as composite quality indices were used to judge the level of quality. The sum of structure, process and outcome value of less than $80 \%$ were judged as poor quality delivery service while $80 \%$ and above were judged as good quality delivery service. Finally qualitative findings were categorized on key thematic area to supplement quantitative findings.

\subsection{Ethical Consideration}

Ethical clearance was obtained from Jimma University ethical review board. Informed (verbal) consent was obtained from the study participants. To maximize validity of responses, staffs were not present during data collection.

\subsection{Operational Definitions and Definition of Terms}

1. Good quality delivery service- the sum of weighted indices of structure, process and outcome items of 80 $100 \%$ was considered as good quality delivery service.

2. Minimum standard for drug- availability of $>80 \%$ of drugs like diclofenac, diazepam, magnesium sulphate, methyldopa/hydralazine/nifedipine, ferrous sulphate, 
vitamin $\mathrm{k}$ injection, ampcline/ amoxicillin, metrondazole tab/inj, TTC eye ointment, ARV drugs (AZT, neverapin), Anti D immunoglobulin, normal saline, ringer lactate, $5 \%$ glucose $/ \mathrm{DW}$, distill water, quinine, ferrous sulfate, anesthesia general/spinal, CAF injection/tablet, paracetamol tablet, pethidine injection, oxytocin (ergometrin), calcium gluconate, vaccines (BCG, polio, TT etc), gentamycin injection, cloxacillin tablet, ceftriaxone injection were considered as good.

3. Minimum standard for equipment- availability of $>80 \%$ equipment like adult weighing scale, thermometer, BP cuff, IV stand, kit for episiotomy, trolley, tap measure, aspiration apparatus, newborn weight scale, stethoscope, refrigerator, fetoscope, examination lights, stretchers, vacuum, forceps, delivery kit, bulb suction, watch, sterile clamp, overhead heater, oxygen cylinder, episiotomy scissors, examination coach, bed were considered as good.

4. Minimum standard for infection prevention materialsavailability of $>80 \%$ infection prevention materials like autoclave/boiling and steaming, bar soap/liquid soap, personal towel, hand lotions, running water, plastic apron, protective foot wear, protective eye wear, elbowlength gloves, safety box/sharp containers, medical waste bins, placenta pit, incinerator, poster and charts relevant to infection control were considered as good.

5. Minimum standard for supply-availability of $>80 \%$ of supplies like HIV kit, blood group reagent, Hgb test, silk/chromic cut gut, plaster/tape, syringe with needle, spinal needle, suture needles, VDRL reagent, surgical glove, disposable glove, gauze, cotton, butterfly needle, cannula, catheter, registration books, obstetric report format, partograph paper, urine dipstick were considered as good.

6. Poor quality delivery service- the sum of weighted indices of structure, process and outcome items less than $80 \%$ was considered as poor quality delivery service.

7. Process- refers to infection prevention practice, client provider interaction and proper use of partograph by using record review of interviewed postnatal mother.

Quality- quality is a multidimensional concept, but in this study, measured in terms of structure, process and outcome for delivery care service provision.

8. Standard partograph measurement- standard protocols were defined based on the time interval as follows:

a) Cervical dilatation, molding, descent of the presenting part and blood pressure monitored every four hours;

b) Fetal heart rate, maternal pulse and uterine contractions monitored every 30 minutes;

c) Condition of the baby after birth should always be recorded on the card.

d) Records not meeting any one of the protocol standards or with parts misplaced/missing or inadequate for each parameter of the partograph will judged as substandard.

9. Structure- refers to the conditions under which care is provided. Structural attributes in this study included; human resources (number, variety, qualification of professionals), material resources (infrastructure, drugs, equipment and supplies), cost for services and transportation.

\section{Result}

\subsection{Infra-Structure}

Five out of nine health facilities had separate waiting room while seven out of nine had postnatal room. Six out of nine had clean toilet and placenta pit. Also five facilities fulfill availability of separate and clean rooms. But only 3 fulfilled the minimum standard of the human resources. Two of them had their own ambulances but the rest used one ambulance with share for transporting laboring mothers. Only one health facility had landline telephones in the delivery ward for emergency call. Five facilities fulfilled the minimum standard of supplies and four health facilities fulfilled the minimum standard for drugs. It was supplemented by majority of the key informants. The respondents said that they did not get all requested drug \& supplies from pharmaceutical fund and supply agency. Out of nine, five facilities fulfilled more than or equals to $80 \%$ of the minimum standard for equipment, but only two facilities fulfilled the minimum standard of infection prevention materials. All facilities refer client with transport accompanied by health care provider and all health facilities were supervised before two months.

\subsection{Process}

Partograph was recorded for $140(54.7 \%)$ of mothers and only $21(8.2 \%)$ of it was recorded according to WHO standard. Among the parameters of maternal condition, 95 $(67.9 \%)$ cervical dilatation and $33(23.6 \%)$ decent were recorded as WHO standard. Seventy nine $(30.9 \%)$ of the respondents were observed interacting with the health provider. Among them privacy was maintained for 60 (75.9\%) and greeting by the health care provider were given for $62(78.5 \%)$. Only two public health facilities were practicing the minimum components of infection prevention practice. This was supplemented by in-depth interview such that only two facilities' staff (3 staffs in number) took infection prevention in service or refreshment training but the rest did not take training.

\subsection{Outcome}

A total of 256 women who gave birth in public health facilities of Arba Minch town and surrounding district were interviewed giving a response rate of $98.1 \%$. The overall clients' satisfaction towards delivery care service after factors analysis was $90.2 \%$. 


\subsection{Quality of Delivery Service}

The overall quality of delivery service among public health facilities in Arba Minch district was $54.06 \%$. This was calculated after assigning the relative weight for structure $(36 \%)$, for process $(34 \%)$ and for outcome $(30 \%)$ and the percent of those fulfilling the minimum requirement were considered and calculated. (Table 1).

Table 1. Assigned relative weights and the calculated weight of quality components in public health facilities of Arba Minch district, Gamo Gofa zone, Southern Ethiopia, April, 2014.

\begin{tabular}{|c|c|c|c|c|}
\hline \multirow{2}{*}{ Dimensions } & \multirow{2}{*}{ Relative weight assigned (\%) } & \multicolumn{2}{|c|}{ Facilities Fulfill minimum requirement } & \multirow{2}{*}{ Weight (\%) } \\
\hline & & Number & Percent & \\
\hline Human resource & 7 & 3 & 33.3 & 2.3 \\
\hline Transportation & 3 & 3 & 33.3 & 1.0 \\
\hline Infrastructures & 6 & 5 & 55.6 & 3.3 \\
\hline Supplies & 5 & 5 & 55.6 & 2.8 \\
\hline Equipment & 6 & 5 & 55.6 & 3.3 \\
\hline Drugs & 5 & 4 & 44.4 & 2.2 \\
\hline Proper use of partograph & 10 & - & 8.2 & 0.8 \\
\hline Client provider interaction & 14 & - & 62.0 & 8.7 \\
\hline Infection prevention practices & 10 & 2 & 22.2 & 2.2 \\
\hline Client satisfaction & 30 & - & 90.2 & 27.06 \\
\hline Total & 100 & & & 54.06 \\
\hline
\end{tabular}

\section{Discussion}

This study assessed the status of quality delivery service at public health facilities of Arba Minch districts using structural capacity, client provider interaction, use of partograph, infection prevention practice, client provider interaction and client satisfaction. Some facilities were facing shortage of rooms to provide proper care and also most of them have shortage of human resource, basic medical equipment, drugs and supplies which compromised quality of care in the studied facilities. This was in line with studies conducted in Uganda, Albania, Sub-Saharan Africa and South Asia [7, 11, 30-32]. It was also consistent with studies conducted in different part of Ethiopia such that poor quality care was fueled by lack of infrastructure and shortage of human resource [13]. Other study conducted in Jimma zone health facilities in 2011 showed that there was shortage of major equipment for maternal and child care in the district hospital [14]. However, this finding was inconsistent with study conducted in 18 different hospitals of Ethiopia such that more than $80 \%$ of drugs and supplies were fulfilled [15]. This difference was probably due to the study setting i.e. the later was conducted in hospital only. Our study also revealed that high proportions of labor progress were not recorded according to modified WHO partograph monitoring. Thus only $8.2 \%$ of women's were followed their labor according to modified WHO partograph and among the recorded partograph only $15 \%$ were complete. This was comparable with a study conducted in Ethiopian hospitals such that 13\% of the parthograph were recorded completely [15]. However, it was lower than studies conducted in Addis Ababa (which was $<40 \%$ ) [21]. It might be due to highly experienced staff and adequate number of health professional in cities than rural part of Ethiopia. Most components of client provider interaction were above $75 \%$ which was different from study conducted in Albania, Turkmenistan and Kazakhstan [31], inadequate attention paid to privacy and confidentiality of laboring mothers. This difference might be due to difference in study setting (The Albania's study was conducted in hospitals with high case load compared to health center but our study includes both hospital and health centers). Our study revealed that providers performed physical examinations for $83.5 \%$ of laboring mothers. This was comparable with study conducted in rural Bangladesh in which $81.3 \%$ of patients during maternal care services were examined [33]. This study also revealed that $78.5 \%$ of pregnant women were given greeting during intrapartum care but a study conducted in 18 Ethiopian hospitals showed that $53 \%$ of pregnant women were greeted [15]. This difference might be due to difference in study setting such that the later study was conducted only in hospitals since high case load in hospital might lead to increase work load for staffs and this in turn might reduce women friendly care. This study also showed that 2 out of 9 facilities were practicing the infection prevention components properly. This was higher than study conducted in Southern Nigeria (which was 13\%) [32]. This difference might be due to sample or facility number difference and area difference. Regarding written procedures and guidelines, four out of 9 facilities owned it. This was higher than study conducted in India (which was 5\%) [34]; and this was probably due to recent training on infection prevention in Ethiopia and might be due to sample or facility number difference. The proportion of mothers who were satisfied towards delivery service in this study was $90.2 \%$. It was almost comparable with study conducted in Wolaita (which was $82.9 \%$ ) [22], but was higher than study conducted in Amhara region (which was 61.9\%) [23]. This difference might be due to study setting and methodological difference, that is, we used $>80$ percent as a cut off point for satisfaction but both studies used $>75$ percent as a cut of point. In this study the quality of delivery service was much lower than the national acceptable figure proposed by performance and quality improvement standard, 2012. It gave recognition for those fulfilling $80 \%$ of the standard but our finding revealed only $54.06 \%$ [35]. This was in line with 
the study conducted in 18 Ethiopian hospitals [15] but this study did not use composite indices to conclude poor delivery quality.

\section{Conclusion}

Overall quality of delivery service fallen far below the recommended standards. Poor quality was observed at all health facilities. Shortage of skilled human resource, infrastructure, drugs, supplies, equipment, infection prevention materials and means of transportation were observed in most health facilities. Unrecorded and very low correct partograph recording were observed and also much lower infection prevention practices were observed but relatively good client provider interactions was observed in this study.

\section{Acknowledgements}

We would like to extend our deepest gratitude to the Jimma University College of Public Health and Medical Sciences, Department of Population and Family Health instructors for their support and constructive comment. Our appreciation goes to the data collectors and supervisors. Lastly, our special thanks go to mothers who participated in the study.

\section{References}

[1] Donabedian A. Evaluating the Quality of Medical Care: the Milbank Quarterly. Milbank Memorial Fund, 2005; 83 (4): 691-729.

[2] Institute of Medicine, Medicare: A Strategy for Quality Assurance. 1990; available at http://www.iom.edu/CMS/8089.aspx.

[3] Maxwell RJ. Quality assessment in health. Br Med J; 1984, 288 (6428): 1470-1472.

[4] Bell and Avan. Developing a framework and instrument to assess the quality of delivery care: a work in progress. Executive Summary, $2010 \quad$ (5). Available at http://www.immpact-international.org.

[5] FMOH, Health Sector Development Program IV, 2010, Ethiopia Federal Ministry of Health.

[6] WHO, UNICEF, UNFPA, WORLD BANK: Trends in Maternal Mortality: 1990 to 2010. 2012, World Health Organization.

[7] Filippi V, Ronsmans C, et al. Maternal health in poor countries: the broader context and a call for action. Lancet, 2006; 368: 1535-1541

[8] Rahman K, Sarkar P, Situation of maternal health care services in Bangladesh. The Social Sciences, 2009; 4 (5): 503.

[9] UN, the Millennium Development Goal Report. 2011. United Nations.

[10] Lilian T, Karen M, Abu M, Bjorg E and Thecla W. Why give birth in health facility? Users' and providers' accounts of poor quality of birth care in Tanzania, BMC, 2013; 13 (174).
[11] Maman D and Pierre F. Human resources and the quality of emergency obstetric care in developing countries: a systematic review of the literature. BMC, 2009; 7 (7).

[12] Jonathan M, Jonathan R, Stuart L, Priya D, and Atul A. Access to essential technologies for safe childbirth: a survey of health workers in Africa and Asia. BMC, 2013; 13 (43).

[13] FGAE. Assessment of Sexual and Reproductive Health, and HIV Linkages. Ethiopia country assessment, 2011. Ethiopia Family Guidance Association.

[14] Beyene W, Jira C, Sudhakar M, Assessment of Quality of Health Care in Jimma Zone, Southwest Ethiopia. Ethiop J Health Sci., 2011; 21.

[15] USAID, MCHIP, Quality of Care for Prevention and Management of Common Maternal and Newborn Complications: A Study of Ethiopia's Hospitals. 2011. Available at www.jhpiego.org.

[16] Mozlan J and Ortayli N. Social science Research initiative on quality of Care. An evaluation of quality of maternity care in three Istanbul updated January.

[17] WHO, Making Pregnancy Safer: The Critical Role of the Skilled Attendant. 2004, World Health Organization.

[18] WHO, Beyond the Numbers: Reviewing Maternal Deaths and Complications to Make Pregnancy Safer. 2004, World Health Organization.

[19] FMOH, National reproductive health strategy, 2006 - 2015. Ethiopia Federal Ministry of Health.

[20] Democratic Republic of Ethiopia/Population Census Commission (FDRE/PCC) (2008), Summary and Statistical Report of the 2007 Population and Housing Census: Population Size by Age and Sex, FDRE/PCC, Addis Ababa.

[21] Engida Y, Berhanu D, Ayalew A and Nebreed F. Completion of the modified WHO partograph during labor in public health institutions of Addis Ababa, Ethiopia. Reproductive Health, 2013; 10 (230)

[22] Bereket Y, Mulat T and Wondimagegn P. Mothers' Utilization of Antenatal Care and their Satisfaction with Delivery Services in Selected Public Health Facilities of Wolaita Zone, Southern Ethiopia. International journal of scientific \& technology research, 2013; 2 (2).

[23] Azmeraw T, Desalegn Z and Yigzaw K: Mothers' satisfaction with referral hospital delivery service in Amhara Region, Ethiopia. BMC, 2011; 11 (78).

[24] Studd J, Partograms and Nomograms of Cervical Dilatation in Management of Primigravid Labor. BM J, 1973; 4 (5890): 451-55.

[25] Surekha T and Pooja J, The impact of use of modified WHO partograph on maternal and prenatal outcome. IJBAR, 2012; 3 (4).

[26] Harriott EM, Williams TV, Peterson MR. Childbearing in US military hospitals: dimensions of care affecting women's perceptions of quality and satisfaction. Birth, 2005; 32: (4).

[27] Bazant ES, Koenig MA, Women's satisfaction with delivery care in Nairobi's informal settlements. International Journal for Quality in Health Care, 2009; 21 (2): 79-86. 
[28] Delvaux T. Quality of normal delivery care in Côted'Ivoire. African Journal of Reproductive Health, 2007; 11 (1).

[29] Liz C. Creel, Justine V. Sass, and Nancy V. Yinger, ClientCentered Quality: Clients' Perspectives and Barriers to Receiving Care Population Council and Population Reference Bureau New Perspectives on Quality of Care: No. 2, Population Council and Population Reference Bureau.

[30] Kiguli J, Elizabeth E, Okui O, Mutebi A, Hayley M and William G. Increasing access to quality health care for the poor: Community perceptions on quality care in Uganda. Patient Preference and Adherence, 2009; 3: 77-85.

[31] Tamburlini G, Siupsinskas G, Bacci A, Quality of Maternal and Neonatal Care in Albania, Turkmenistan and Kazakhstan: A Systematic, Standard-Based, Participatory Assessment. PLoS ONE 6 (12): 28763.
[32] Okonofua F, Okpokunu E, Aigbogun O, Nwandu C, Mokwenye $\mathrm{C}$ and Kanguru L, et al. Assessment of infection control practices in maternity units in Southern Nigeria. International Journal for Quality in Health Care, 2012: 1-7.

[33] Jorge Mendoza Aldana, Helga Piechulek, Ahmed Al-Sabir. Client satisfaction and quality of health care in rural Bangladesh. Bulletin of the World Health Organization, 2001; 79 (6).

[34] Rajesh M, Dileep VM, KV Ramani, Sheetal S and Julia H. Infection control in delivery care units, Gujarat state, India: A needs assessment. BMC, 2011; 11 (37).

[35] JHPIEGO: Performance and quality improvement standards for EMNC health center/hospital intrapartum care \& infection prevention, February, 2012 draft Ethiopia, version 1. 